\title{
Bone Regenerative Medicine and Bone Grafting
}

\section{Harsini SM* and Tafti AK}

Department of Pathology, School of Veterinary Medicine, Shiraz University, Iran

*Corresponding author: Somayeh Monazzah Harsini, DVM, PhD Department of Pathology, School of Veterinary Medicine, Shiraz University, Shiraz, Iran, Tel: 09188369120; Email: somayehmonazah@gmail.com

\section{Mini Review \\ Volume 3 Issue 4}

Received Date: October 19, 2018

Published Date: December 04, 2018

DOI: $10.23880 /$ oajvsr-16000167

\section{Abstract}

Bone tissues can repair and regenerate it: in many clinical cases, bone fractures repair without scar formation. Nevertheless, in large bone defects and pathological fractures, bone healing fail to heal. Bone grafting is defined as implantation of material which promotes fracture healing, through osteoconduction osteogenesis, and osteoinduction. Ideal bone grafting depends on several factors such as defect size, ethical issues, biomechanical characteristics, tissue viability, shape and volume, associated complications, cost, graft size, graft handling, and biological characteristics. The materials that are used as bone graft can be divided into separate major categories, such as autografts, allografts, and xenografts. Synthetic substitutes and tissue-engineered biomaterials are other options. Each of these instances has some advantages and disadvantages. Between the all strategies for improving fracture healing and enhance the outcome of unification of the grafts, tissue engineering is a suitable option. A desirable tissue-engineered bone must have properties similar to those of autografts without their limitations. None of the used bone grafts has all the ideal properties including low donor morbidity, long shelf life, efficient cost, biological safety, no size restriction, and osteoconductive, osteoinductive, osteogenic, and angiogenic properties; but Tissue engineering tries to supply most of these features. In addition it is able to induce healing and reconstruction of bone defects. Combining the basis of orthopedic surgery with knowledge from different sciences like materials science, biology, chemistry, physics, and engineering can overcome the limitations of current therapies. Combining the basis of orthopedic surgery with knowledge from different sciences like materials science, biology, chemistry, physics, and engineering can overcome the limitations of current therapies.

Keywords: Bone grafting; Osteoinduction; Osteoconduction osteogenesis; Cancellous; Autografts

\section{Introduction}

We know that the bone tissues can repair and regenerate itself: in many clinical cases, bone fractures repair without scar formation. Nevertheless, in large bone defects and pathological fractures, bone healing fail to heal. Also infection of the bone or insufficient blood supply can influence bone healing negatively, that cause delayed unions or even non-unions [1]. After blood, Bone is the second most commonly transplanted tissue. Bone 
grafting is defined as implantation of material which promotes fracture healing, through osteoconduction osteogenesis, and osteoinduction [2].

Ideal bone grafting depends on several factors such as defect size, ethical issues, biomechanical characteristics, tissue viability, shape and volume, associated complications, cost, graft size, graft handling, and biological characteristics [3].

The materials that are used as bone graft can be divided into separate major categories, such as autografts, allografts, and xenografts. Synthetic substitutes and tissue-engineered biomaterials are other options. Each of these instances has some advantages and disadvantages. Allografting and xenografting are with osteoconductive and osteoinductive characteristics but without the osteogenic properties of autografting [4]. Autogenous bones are the "gold standard" in regeneration of small bone defects because they have strong osteogenic properties for bone healing. Some of the autografts disadvantages are donor site morbidity and major vessel injuries during harvesting. Because of these reasons, some alternative options have introduced [5]. Allograft is another option with risk of rejection and transmission of diseases. Allografts have less incorporating characteristic with host tissues in comparing with autografts. Xenografts, moreover to allografts disadvantages, have risks of transmission of zoonotic diseases. Because of these problems, bone tissue engineering has been introduced recently [6]. Bone tissue engineering involves application of scaffolds, growth factors and cells. Using tissue engineering techniques decrease disadvantages of traditional bone grafting and improve osteoinductivity, osteoconductivity, and osteogenicity [7] (Figure 1).

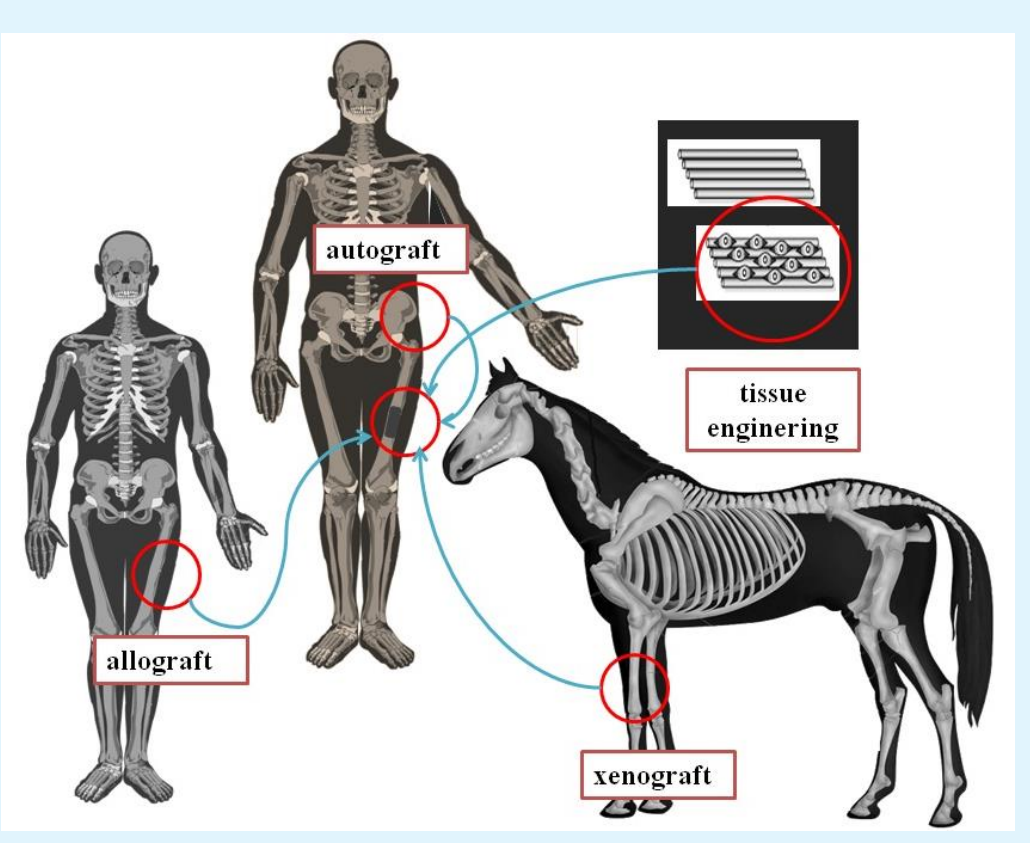

Figure 1: All type of bone grafting, autograft, allograft, xenograft and tissue engineering.

Tissue engineering has several limitations, such as: use of an extent variety of substance in producing tissueengineered scaffolds or grafts. Therefore, translational examinations of each material are limited, that reduce their clinical applications. So, some important facet of interaction of host graft and immune response to these biomaterials, and scaffolds are still not clear. Tissue engineering advances make us the able to repair bone tissue [8].

\section{Bone Grafts Structure}

Cortical bone usually has higher mineral contents in comparing to cancellous bone. Besides, presence of spaces in cancellous bone, make it more osteogenic in comparing cortical bone. Strength and stiffness of the cortical bone are more than cancellous bone. Surgeon must be aware of these structural differences in bone in selecting a graft. Bone grafts may be cancellous, cortical, or corticocancellous [9]. 
Cortical bone grafting mostly are used for strength and structural support, and cancellous bone grafting for osteogenesis. Using cortico-cancellous bone grafts supply osteogenesis and structural support. Cancellous bone grafting is usually used in small bone defects, fracture non-union, maxillofacial defects, spinal fusion, and dental defects. Cancellous bone grafts lack mechanical strength. Cancellous bone grafts has porous structure and enhance bony ingrowth because it allows faster revascularization [10].

\section{Properties of Bone Grafts}

Comprehending of the biological properties of bone grafts is needed to decide what kind of bone graft is more suitable for a given condition. An ideal bone graft material should have osteoconductivity, osteoinductivity, and osteogenesis characteristics [11].

Osteoconduction is a property that in accordance with the graft acts as a resorbable and permanent scaffold, mechanically supporting for vessels ingrowth. These properties initiate new bone formation [12].

Osteoinduction is the ability of the bone graft for inducing bone formation with differentiation stem cells from the surrounding tissues for producing osteoprogenitor cells and development of osteoblasts. This ability has discovered in different growth factors such as bone morphogenetic proteins including transforming growth factor- $\beta$, BMP- 2 and BMP-7, insulinlike growth factor, platelet-derived growth factor and fibroblast growth factor [13].

Osteogenesis is function to produce new bone formation by the osteoblastic differentiation from osteoprogenitor cells present in host bone or graft material. This characterization is present in autografts in comparing to allografts or xenografts, due to the cellular content of allografts and xenografts have low viability in host body [14].

Among all bone grafts, just autografts possess all three above features and Allografts and xenografts exhibit just one or two of the three features of an ideal bone graft [15].

\section{Unification of Bone Grafts}

Unification of grafts within the host bone depends on properties like graft revascularization. Fast unification and good healing of the bone graft can be achieved with optimal quality and speed of revascularization, if there be sufficient independent vascular supply for defect site. In non-vascularized bone grafts, capillaries slowly penetrate into the graft, and the healing process is thus prolonged [16].

In any case of the structure or source, all transplanted grafts continued through five stages: inflammation, revascularization, osteoinduction, osteoconduction, and finally remodeling. The duration of each stage can vary depending on the properties of the bone graft. Incorporation will delay if interference with vascularization, including excessive micromotion and infections at the reconstructed area [17]. In cortical bone grafts, vascularization or capilarization is slower and occurs along Haversian canals, despite its performed by sneaking substitution in cancellous bone grafts. In the last process, the new osteoblasts line the trabeculae of new bone to resorption of bone by osteoclasts; but in cortical grafts, osteoclastic resorption is a prerequisite before giant osteoblasts can produce new bone formation [18].

In second and third stages of bone healing, the immune system of host becomes active against the donor antigenicity. Unification of cancellous autogenous bone is fastest, followed by cortical autogenous bone, cancellous and cortical allogenous, and xenogenous, respectively. Because allogenous and xenogenous bone are not genetically matched, they would initiate an immune response. When allogenous and xenogenous bone are used, so, it's more likely that the bone graft will fail and the donor tissue will rejected. Fresh-frozen or freezedried allografts and xenografts weaker immunologic responses than fresh ones [19].

\section{Types of Grafts}

Autografts, allografts, and xenografts as well as bone graft substitutes are all applied to enhance bone defects healing. Autografts bone has limitations in massive bone defects and pathologic fractures; so, other grafts have introduced to overcome these autografts limitations. All types of the available options have limitations and selection of a good graft depends on the surgeon's experience [20].

\section{Autografts}

Bone grafts which are harvested from one site of body and implanted into other site in the same person are termed autogenous bone grafts, autologous, or autografts. Autografts may be cortical or cancellous bone or corticocancellous grafts. Fresh autogenous bone contains surviving cells and also osteoinductive proteins including 
BMPs. They are the best materials that are available, because of lacking immunogenicity [21]. They keep their viability short time after transplantation and the lack of immunogenicity so they enhance the chances of graft unification into the host site. In addition, the osteoinductive, osteoconductive, and osteogenic properties of autografts are optimal, and give us the presence of osteogenic cells, osteoprogenitor cells, stem cells and growth factors [22]. Autografts don't transmit viral disease; in addition, they offer structural support for implanting devices and, eventually, become mechanically efficient structures as they are unified into the host bone through creeping substitution. Autografts implies supplemental surgery with a high chance of donor site morbidity, pain, and complications. If in the orthopedic surgery we need massive grafting, sufficient amounts of autogenous bone may not be available [23].

Different sites of body have been applied for harvesting the bone grafts. Grafts may be harvested from the proximal or distal radius and tibia, the proximal humerus, calcaneus, iliac crest, the distal ulna, ribs, and the proximal olecranon [24]. Each of these sources has advantages and also disadvantages; but the iliac crest has most advantages, including: easy accessibility and availability of adequate amounts cortical and cancellous bones. However, graft obtained from this site can make urethral, arterial, nerval, and injury, and pelvic fracture. So, other sites like distal radius have been used [25].

Generally, the success of bone autograft depends on the survival of the osteogenic cells, handling of the graft, conditions at the recipient bed, type of graft chosen. Fresh autograft has the capability of new bone formation supporting. If inductive molecules like BMPs be locally delivered on a graft, the stem cells can be attracted to the site and they are capable of reproducibly inducing new bone formation [26].

\section{Allografts}

Allografts are optioned from one person and implanted into another person from the same species. Because of the autografts limitations, allografts have used clinically as a common alternative to autografts. Allograft bones are finded from regional tissue banks and by most major spinal and orthopedic companies [27].

Allografts are applied in both structural and morselized forms and are supplied as cortical, cancellous, or cortico-cancellous bone grafts and in different forms such as powder, cancellous cubes, and cortical chips. Allografts also can be processed in the forms of fresh, fresh-frozen, or freeze-dried forms, mineralized or demineralized. They can be achieved from cadavers or living persons [28]. The most important advantages of them are their ready availability in different sizes and shapes, avoidance of sacrificing host tissues, and absents of challenges of donor site morbidity. The Allogenous bone has variable osteoconductive and osteoinductive properties but lack viable cells and, so, have no osteogenic potential [29].

The allografts have the risk of transmitting bacterial and viral diseases, including: hepatitis B and C, HIV and in addition they can induce immunological reactions which interfere with the fracture healing process and lead to bone graft rejection. Moreover, the rate of bone healing, in application of allografts, is usually lower than the autografts [30].

Because of the higher chance of immune response and also the risk of viral and bacterial transmission, fresh bone allografts are rarely used; and frozen and freezedried allografts, are used which immune reactions is minimized, and the biomechanical and biological properties are only partially affected [31].

Freeze-dried and fresh-frozen bone allografts induce more prompt graft incorporation, vascularization, and bone regeneration than fresh bone allograft. Freeze drying of bone allografts produces a safer bone graft in terms of reducing the risk of immunologic responses and transmission of diseases [32]. Despite modern sterilization, processing of bone allografts with using freeze-drying and fresh-frozen techniques and treating the graft by acetone, ethylene oxide, hypotonic solutions, or gamma irradiation that can eliminate cellular, bacterial and viral particles and therefore decrease the risk of infectious and transmissible diseases, the application of bone allografts is not completely safe [33]. The processes like this can destroy the osteoblasts and denature proteins present in the graft including BMPs and alter osteoinductive and osteoconductive properties, and essentially eliminating the osteogenic characteristic [34].

So, freeze-dried bone allografts take longer to become incorporated and revascularized than autografts. Freezedrying method also decreases the mechanical strength of the alografts, and the cost of process allografts is high. Mineral component of allografts can be removed by acid and process called demineralization to obtain demineralized bone matrix that has osteoconductive and osteoinductive properties [35]. Demineralized bone matrix revascularizes fast, and its biological properties are attributed to proteins and different growth factors 
present in the ECM. Because of these major disadvantages, bone allografts aren't the perfect substitute for autograft [36].

\section{Xenografts}

Another alternative to autografts are xenografts, that known as xenogenic grafts or heterologous. Xenograft bones are harvested from one person and transplanted into another person of a different species. The common attainable bone xenografts are derived from coral, bovine and porcine sources. Xenografts are an unlimited provider of available material for transplantation in humans [24]. A major risk with xenografts is the potential transmission of zoonotic diseases. Xenograft bones, like allograft, lose their osteogenic and some osteoinductive characteristic during the processing. Xenografts make poor clinical outcome [34].

There are several researches about using different animals in xenografting such as canine bovine, porcine and coral graft. Bovine bone xenografts have had organic substances extracted; the remaining structure with fine pores is like natural bone, chemical compound or microstructure. It has a non-antigenic, natural porous matrix and is identical to the mineral phase of bone tissue [24]; it has been demonstrated which high osteoconductive property and to show a very low resorption rate. The researches demonstrated that all the materials, with the exception of mineralized bovine bone [Surgibone], were biocompatible for osteoblasts of human. Anorganic bovine bone doesn't have osteoinductive properties, and when it is in the form of granules makes it difficult to hold on surgical sites. Moreover, bovine xenograft is non resorbable in vivo [37].

\section{Bone Tissue Engineering}

Tissue engineering means combining those branches of scientific fields so that the principles of the life knowledge and engineering are applied to regenerate, restore, maintain, or increase in the quality of tissue structure and tissue function. It's based on the understanding of regeneration and tissue formation, and production of new functional tissues. The tissue engineering scientists hope to reach this purpose by combining knowledge from materials science, physics, chemistry, engineering, medicine, and biology [37]. This question is mooted that what is needed by orthopedic surgeons to properly apply tissue engineering for new bone formation? Bone tissue engineering, like any other tissue healing methodologies, needs the three following essential elements including cells, extracellular matrix, and growth factors: Cellular components must be present to give rise to new structural tissue [38]. They can be obtained from an exogenous source or endogenously from the surrounding tissues. Differentiators and growth factors must be present for the suitable development of the cellular elements. They can be provided exogenously, produce by the transferred cells, or derived from endogenous sources. A good scaffold can be instituted to supply a substrate for cellular proliferation, attachment and differentiation [39]. It may serve to prevent mobilization, provide biomechanical support, organize and align tissue hierarchy and orient growth factors or drugs to the responding cells [40]. Bone has a three dimensional configuration, and the cells don't grow in a three dimension fashion in vitro, therefore a scaffold as a three dimensional structure, mimicking bone structure, must be used so that the new tissue can be grown in a three dimension manner [41].

\section{Discussion and Conclusion}

To design an efficient bone graft and substitute, the orthopedic surgeons and researchers must have sufficient knowledge of the properties of grafts including osteoconductivity, osteoinductivity, and osteogenesis, and their advantages and disadvantages [22]. Autografts usually are the gold standard for bone regeneration. Between the all strategies for improving fracture healing and enhance the outcome of unification of the grafts, tissue engineering is a suitable option. A desirable tissueengineered bone must have properties similar to those of autografts without their limitations [16].

None of the used bone grafts has all the ideal properties including low donor morbidity, long shelf life, efficient cost, biological safety, no size restriction, and osteoconductive, osteoinductive, osteogenic, and angiogenic properties [6]; but Tissue engineering tries to supply most of these features. In addition it is able to induce healing and reconstruction of bone defects. Combining the basis of orthopedic surgery with knowledge from different sciences like materials science, biology, chemistry, physics, and engineering can overcome the limitations of current therapies [41].

\section{References}

1. Liu M, Yu X, Huang F, Cen S, Zhong G, et al. (2013) Tissue engineering stratified scaffolds for articular cartilage and subchondral bone defects repair. Orthopedics 36(11): 868-873.

2. Gupta A, Woods MD, Illingworth KD, Niemeier R, Schafer I, et al. (2013) Single walled carbon nanotube 


\section{Open Access Journal of Veterinary Science \& Research}

composites for bone tissue engineering. J Orthop Res 31(9): 1374-1381.

3. Zugravu MV, Smith RA, Reves BT, Jennings JA, Cooper JO, et al. (2013) Physical properties and in vitro evaluation of collagen chitosan- calcium phosphate microparticle-based scaffolds for bone tissue regeneration. J Biomater Appl 28(4): 566-579.

4. Monazzah Harsini S, Oryan A (2018) Growth Factor of Platelet-Rich Plasma and its Application in Trauma and Orthopedic Surgery. EC Orthopaedics 9: 139-146.

5. Amruthwar SS, Janorkar AV (2013) In vitro evaluation of elastin-like polypeptide collagen composite scaffold for bone tissue engineering. Dent Mater 29(2): 211-220.

6. Faldini C, Miscione MT, Acri F, Chehrassan M, Bonomo M, et al. (2011) Use of homologous bone graft in the treatment of aseptic forearm nonunion. Musculoskelet Surg 95(1): 31-35.

7. Patel H, Bonde M, Srinivasan G (2011) Biodegradable polymer scaffold for tissue engineering. Trends Biomater Artif Organs 25(1): 20-29.

8. Mahato NK (2011) Characterization of corticocancellous bone along the iliac crest: focus on graft harvesting. Surg Radiol Anat 33(5): 433-437.

9. Yamada S, Nagaoka H, Terajima M, Tsuda N, Hayashi Y, et al. (2013) Effects of fish collagen peptides on collagen post-translational modifications and mineralization in an osteoblastic cell culture system. Dent Mater J 32(1): 88-95.

10. Hegde C, Shetty V, Wasnik S, Ahammed I, Shetty V (2012) Use of bone graft substitute in the treatment for distal radius fractures in elderly. Eur J Orthop Surg Traumatol 23(6): 651-656.

11. Bigham-Sadegh A, Karimi I, Alebouye M, ShafieSarvestani Z, Oryan A (2013) Evaluation of bone healing in canine tibial defects filled with cortical autograft, commercial-DBM, calf fetal DBM, omentum and omentum-calf fetal DBM. J Vet Sci 14(3): 337-343.

12. Zimmermann G, Moghaddam A (2011) Allograft bone matrix versus synthetic bone graft substitutes. Injury 42(2): S16-S21.

13. Oryan A, Monazzah S, Bigham-Sadegh A (2018) The Effects of Melatonin in Bone Healing. Vet Sci Res 3(2): 000155.

Harsini SM and Tafti AK. Bone Regenerative Medicine and Bone Grafting. Vet Sci Res 2018, 3(4): 000167.
14. Folsch C, Mittelmeier W, Bilderbeek U, Timmesfeld N, von Garrel T, et al. (2012) Effect of storage temperature on allograft bone. Transfus Med Hemother 39(1): 36-40.

15. Scaglione M, Fabbri L, Dell'omo D, Gambini F, Guido G (2013) Long bone nonunions treated with autologous concentrated bone marrow-derived cells combined with dried bone allograft. Musculoskelet Surg 98(2): 101-106.

16. Ohba S, Tei Chung YU (2013) Bone and cartilage diseases and regeneration. Identification of osteogenic signal and the development of artificial bones. Clin Calcium 23(12): 1723-1729.

17. Oryan A, Bigham-Sadegh A, Monazzah S (2016) Fish bone versus fish demineralized bonematrix (vertebra) effects on healing of experimental radial defect in rat model. Comp Clin Pathol 14(2): 981-985.

18. Aravamudhan A, Ramos DM, Nip J, Harmon MD, James $\mathrm{R}$, et al. (2013) Cellulose and collagen derived micronano structured scaffolds for bone tissue engineering. J Biomed Nanotechnol 9(4): 719-731.

19. Lee M, Song HK, Yang KH (2012) Clinical outcomes of autogenous cancellous bone grafts obtained through the portal for tibial nailing. Injury 43(7): 1118-1123.

20. Muller MA, Frank A, Briel M, Valderrabano V, Vavken P, et al. (2012) Substitutes of structural and nonstructural autologous bone grafts in hind foot arthrodeses and osteotomies: a systematic review. BMC Musculoskelet Disord 14: 59.

21. Garg B, Goyal T, Kotwal PP, Sankineani SR (2012) Local distal radius bone graft versus iliac crest bone graft for scaphoid nonunion: a comparative study. Musculoskelet Surg 97(2): 109-114.

22. Lee SS, Huang BJ, Kaltz SR, Sur S, Newcomb CJ, et al. (2013) Bone regeneration with low dose BMP-2 amplified by biomimetic supramolecular nanofibers within collagen scaffolds. Biomaterials 34(2): 452459.

23. Pastorino L, Dellacasa E, Scaglione S, Giulianelli M, Sbrana F (2013) Oriented collagen nanocoatings for tissue engineering. Colloids Surf B 114: 372-378.

24. Ma J, Both SK, Yang F, Cui FZ, Pan J, et al. (2014) Concise review: cell-based strategies in bone tissue engineering and regenerative medicine. Stem Cells Transl Med 3(1): 98-107. 


\section{Open Access Journal of Veterinary Science \& Research}

25. Athanasiou VT, Papachristou DJ, Panagopoulos A, Saridis A, Scopa CD, et al. (2010) Histological comparison of autograft, allograft-DBM, xenograft, and synthetic grafts in a trabecular bone defect: an experimental study in rabbits. Med Sci Monit 16(1): BR24-BR31.

26. Mauffrey C, Madsen M, Bowles RJ, Seligson D (2012) Bone graft harvest site options in orthopaedic trauma: a prospective in vivo quantification study. Injury 43(3): 323-326.

27. Janicki P, Schmidmaier G (2011) What should be the characteristics of the ideal bone graft substitute? Combining scaffolds with growth factors and/or stem cells. Injury 42(2): S77-S81.

28. Oryan A, Bigham-Sadegh A, Monazzah S (2017) Characterization of turtle demineralized bone matrix (DBM) and turtle bone in rat radial bone defects; radiological, histopathological, biomechanical and scanning electron microscopic evaluation. Veterinarski Arhiv 87(4): 523-534.

29. Moshiri A, Oryan A (2012) Role of tissue engineering in tendon reconstructive surgery and regenerative medicine: current concepts, approaches and concerns. Hard Tissue 1: 11.

30. Yazar S (2010) Onlay bone grafts in head and neck reconstruction. Semin Plast Surg 24: 255-261.

31. Dimitriou R, Jones E, McGonagle D, Giannoudis PV (2011) Bone regeneration: current concepts and future directions. BMC Med 9: 66.

32. Monazzah S, Oryan A, Bigham-Sadegh A, MeimandiParizi A (2017) Application of bovine bone versus bovine DBM graft on bone healing of radial defect in rat. Comp Clin Pathol 4(2): 11-18.

33. Hamilton PT, Jansen MS, Ganesan S, Benson RE, HydeDeruyscher R, et al. (2013) Improved bone morphogenetic protein-2 retention in an injectable collagen matrix using bifunctional peptides. PLoS One 8(8): e70715.
34. Vittayakittipong P, Nurit W, Kirirat P (2012) Proximal tibial bone graft: the volume of cancellous bone, and strength of decancellated tibias by the medial approach. Int J Oral Maxillofac Surg 41(4): 531-536.

35. Bayod J, Becerro-de-Bengoa-Vallejo R, Losa-Iglesias ME, Doblare M (2012) Mechanical stress redistribution in the calcaneus after autologous bone harvesting. J Biomech 45(7): 1219-1226.

36. Mistura DV, Messias AD, Duek EA, Duarte MA (2013) Development, characterization, and cellular adhesion of poly(l-lactic acid)/poly (caprolactonetriol) membranes for potential application in bone tissue regeneration. Artif Organs 37(11): 978-984.

37. Nguyen DT, McCanless JD, Mecwan MM, Noblett AP, Haggard WO, et al. (2013) Balancing mechanical strength with bioactivity in chitosan-calcium phosphate 3D microsphere scaffolds for bone tissue engineering: air- vs. freeze-drying processes. J Biomater Sci Polym Ed 24(9): 1071-1083.

38. Oryan A, Monazzah-Harsini S (2018) Characteristics of the Main Constituents Used in Bone Tissue Engineering. EC Orthopaedics 9: 105-114.

39. Tang Y, Zhao Y, Wang X, Lin T (2014) Layer-by-layer assembly of silica nanoparticles on 3D fibrous scaffolds: enhancement of osteoblast cell adhesion, proliferation and differentiation. J Biomed Mater Res 102(11): 3803-3812.

40. Oryan A, Monazzah S, Bigham-Sadegh A (2015) Bone injury and fracture healing biology Biomed Environ Sci 28(1): 57-71.

41. Yang YL, Chang CH, Huang CC, Kao WM, Liu WC, et al. (2014) Osteogenic activity of nanonized pearl powder/poly (lactide-co-glycolide) composite scaffolds for bone tissue engineering. Biomed Mater Eng 24(1): 979-985. 